P72 (continued)

age group; yet with regards to some lesson contents, educators appeared to be far more sensitive to potential issues than did the teens.

Funding: NJ EFNEP.

\section{P73 Cultivating Connections with Farm to School Activities During School Closures}

Patty Case, MS, RD, patty.case@oregonstate.edu, Oregon State University Extension Service, 6923 Washburn Way, Klamath Falls, OR, 97601; Cheryl Kirk, BS, RD, Oregon State University Extension Service Josephine Count

Objective: To engage youth and their families in a webbased hybrid club for farm to school activities as an alternative to school-based activities when schools closed due to the pandemic.

Use of Theory or Research: Farm to school programs increase children's access to and knowledge of fresh and local foods primarily through experiential learning (Kolb's Experiential Learning Theory) such as gardening, tasting food and field trips.

Target Audience: Youth, grades 2-6, and their families who experienced disruption in usual learning and social activities due to COVID-19 restrictions.

Program Description: Experiential activities are the hallmark of farm to school education. In March 2020 all hands-on, school based activities planned by Extension faculty were cancelled due to the pandemic. Faculty responded by offering garden-cooking clubs using a web-based hybrid approach independent of schools. Faculty facilitated biweekly virtual meetings of 30 minutes for 6-8 weeks for youth and their families. Asynchronous activities were also offered via a website, kits, video demonstrations, farmers market vouchers and when possible, farm tours.

Evaluation Methods: Adult caregivers received a survey at the end of the 6-8 week club sessions to evaluate their child's and family's level of engagement including queries on how much time they spent using web-based tools and how likely they were to start a garden.

Results: One hundred and ninety youth, grades $2-6$, and their families participated in 4 virtual and/or hybrid clubs delivered by Extension faculty from March 2020 to October 2020. Caregiver responses from the survey $(n=46)$ indicated $83 \%$ of families expanded or started a garden. On average youth and/or families spent 47 minutes per week engaged in program activities including online learning.

Conclusion: It appears that virtual programming can actively engage youth and their families in farm to school education and may be a tool to supplement resource intensive hands-on strategies in the future. Further research is need to determine which components are best suited for web-based delivery vs in person delivery.

Funding: Oregon Department of Education.
P74 Diet Behavior During CovID: A Qualitative Study of Former Participants of a Food Security Intervention

Miranda Cook, MPH, macook2@emory.edu, Laney Graduate School, Emory University, 1518 Clifton Rd, Atlanta, GA, 30322; David Denton, BS, Rollins School of Public Health, Emory University; Stacie Schmidt, MD, Emory University School of Medicine; Amy Webb Girard, $P h D$, Hubert Department of Global Health, Emory University

Background: The Georgia Food for Health program $(\mathrm{GF} 4 \mathrm{H})$ is a clinic-based nutrition education and food security intervention program that has demonstrated the ability to improve dietary behaviors. However, little is known about changes in behaviors during COVID-related lockdowns.

Objective: To characterize diet behavior changes experienced by former participants of a food security intervention during COVID-related lockdowns.

Study Design \& Setting: We conducted semi-structured interviews by phone with 17 patient participants in May June 2020 with program participants who had completed the program within the past 6 months. Participants were asked questions about the program, diet and stress-related behaviors, and changes in these behaviors within the COVID lockdown period.

Participants: Participants were mainly middle-aged (mean age 59 years), African American (100\%), women (59\%). Approximately half screened positive for food insecurity $(47.1 \%)$. All participants were healthy at the time of the interview.

Analysis: Transcripts of interviews were analyzed for both apriori and emergent themes using thematic analysis.

Results: We identified 3 central themes. First, participants discussed changes in shopping behaviors to minimize time spent in stores using approaches like shopping lists to plan routes within the store or sending younger relatives to shop for them. Due to supply issues at stores, participants discussed adapting recipes to include available options. Second, participants discussed disinterest in cooking, a lack of motivation to engage in healthy eating strategies they had previously adopted, and anticipation of regretting these behaviors post-COVID. Third, participants were largely uncomfortable at the prospect of resuming in-person programming and preferred virtual options due to safety concerns.

Conclusion: Among former participants of the GF4H program, shopping and cooking behaviors shifted during the COVID-19 lockdown period to adapt to safety guidelines and to overcome supply issues. Motivation to engage in healthy cooking and eating behaviors was diminished. Ongoing program efforts should focus on virtual options to engage and re-engage participants.

Funding: $\mathrm{NIH}$. 